\title{
Analogy-Driven 3D Style Transfer
}

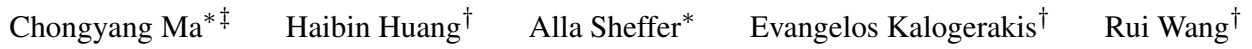 \\ * University of British Columbia $\quad \dagger$ University of Massachusetts Amherst $\quad \ddagger$ University of Southern California
}

\begin{abstract}
Style transfer aims to apply the style of an exemplar model to a target one, while retaining the target's structure. The main challenge in this process is to algorithmically distinguish style from structure, a high-level, potentially ill-posed cognitive task. Inspired by cognitive science research we recast style transfer in terms of shape analogies. In IQ testing, shape analogy queries present the subject with three shapes: source, target and exemplar, and ask them to select an output such that the transformation, or analogy, from the exemplar to the output is similar to that from the source to the target. The logical process involved in identifying the source-to-target analogies implicitly detects the structural differences between the source and target and can be used effectively to facilitate style transfer. Since the exemplar has a similar structure to the source, applying the analogy to the exemplar will provide the output we seek. The main technical challenge we address is to compute the source to target analogies, consistent with human logic. We observe that the typical analogies we look for consist of a small set of simple transformations, which when applied to the exemplar generate a continuous, seamless output model. To assemble a shape analogy, we compute an optimal set of source-to-target transformations, such that the assembled analogy best fits these criteria. The assembled analogy is then applied to the exemplar shape to produce the desired output model. We use the proposed framework to seamlessly transfer a variety of style properties between $2 D$ and $3 D$ objects and demonstrate significant improvements over the state of the art in style transfer. We further show that our framework can be used to successfully complete partial scans with the help of a user provided structural template, coherently propagating scan style across the completed surfaces.
\end{abstract}

Categories and Subject Descriptors (according to ACM CCS): I.3.5 [Computer Graphics]: Computational Geometry and Object Modeling-Curve, surface, solid, and object representations

\section{Introduction}

Given a target and an exemplar shapes, the goal of style transfer is to generate an output model that retains the target's structure, often linked to its functionality, but reflects the style characteristics of the exemplar [XLZ* 10]. In the teaser example (Figure 1), the challenge is to generate an output which retains the structure of the target corner-sofa model, but has the style elements (back, legs and pillow shape) of the exemplar armchair. Algorithmically defining style-structure separation is a context-based, and potentially ambiguous task [MWZ*13]. To relive this ambiguity, in the context of image style transfer, researchers proposed using an input source model which has the same structure as the exemplar and the style of the target [ $\left.\mathrm{HJO}^{*} 01\right]$. The hope is that the source-totarget relationship will provide insights into the structural differences between the exemplar and the target. However, even with this formulation, the computation of a suitable output remains an open problem and existing style-transfer research is largely limited to the 2D setup (Section 2). To enable style transfer between 3D shapes we use insights from
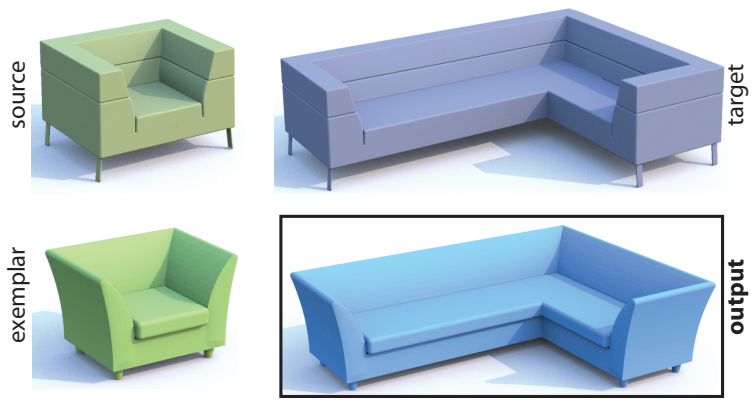

Figure 1: Our algorithm computes the analogy relationship between the source armchair and target sofa (top row) and applies it to the exemplar armchair to synthesize a new corner sofa (bottom row) in the style of the exemplar.

cognitive science and cast style transfer in geometric, or shape, analogy terms.

Shape analogy queries, also referred to as geometric or proportional analogies, are a standard feature in IQ 
tests [BO00,SGKK09]. The typical test input consists of three shapes, a source, a target, and an exemplar. The test subject's goal is to select or construct an output shape such that the same relation, or analogy, holds between the exemplar and the output as between the source and the target (Figure 2).

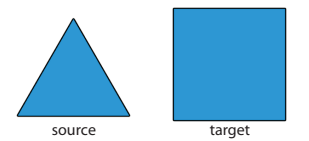

To obtain the output, test subjects are expected to assemble the transformations, or analogies, relating the source to the target,

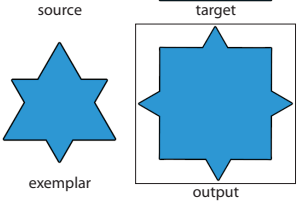

Figure 2: Typical shape analogy query. as well as establish a mapping, or correspondence, between the source and the exemplar. They are then expected to leverage this mapping and apply a similar analogy transformation to the exemplar in order to construct the missing output.

We believe that the analogies humans perceive implicitly define structural differences and thus a framework that mimics analogy query solution can be used to facilitate algorithmic style transfer. There is a fair amount of research on dense shape correspondence which can be adapted to compute source-to-exemplar mappings. Hence the main challenge in applying the analogy metaphor to shape synthesis is to mimic the process by which humans select source-to-target analogies. We speculate that in both IQ tests and style-transfer problems the analogies often consists of several individual partial transforms, e.g., mapping individual triangle edges to those of the square as shown in Figure 2, or matching replicating portions of an armchair's seat and back, translating and rotating them appropriately, to assemble a sofa (Figure 4). Different choices of partial transformations, and the patches that they operate on, would lead to different outputs. We seek the choice that closely matches human logic. Based on literature reviews [Eva68, BO00, SGKK09] and analysis of typical inputs, we speculate that humans compute analogies by mentally segmenting the source into a compact set of patches which map to the target via simple per-patch transformations, i.e., ones that minimally change the patches in an effort to fit the target. In the context of shape synthesis, we additionally prefer analogies that maintain continuity between transformed adjacent patches on the output model.

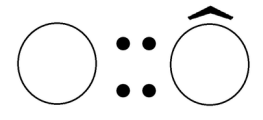

Lastly, in style transfer, as in IQ tests, the target shapes can contain elements with no appropriate source counterpart (see Figure 3). Test

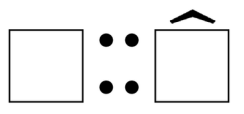
subjects are expected to incorporate these elements as-is into the output. We apply a similar logic to styletransfer data (e.g. see Figure 8).

Figure 3: Selfreferencing

IQ tests are designed to make the analogy. choice of the analogies clear and unambiguous by presenting test subjects with a small set of alternatives. In contrast in the style transfer setup we search within the exponentially large set of all possible patches and per-patch transformations. To make the problem tractable, we restrict the per-patch

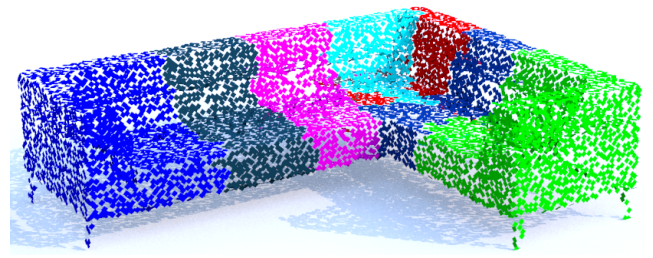

Figure 4: The transformations used to assemble the source to target analogy in Figure 1.

source-to-target transformations that we consider to similarity transformations, i.e., translations, rotations, and scales that map source surface patches to corresponding patches on the target. This assumption is well suited for processing man-made or engineered shapes where both fine details and structural elements are typically related to one another through similarity transforms [WXL*11, LZW*13].

To compute analogies that satisfies our criteria of simplicity, compactness and continuity, we formulate the search for the desired piece-wise transformations as a multi-label optimization problem (Section 5). We apply the resulting piece-wise analogy transformation to the exemplar using a source-to-exemplar mapping computed as described in Section 4. To account for differences in shape between the exemplar and the source, and avoid discontinuities in the output we use non-rigid patch alignment (Section 6).

Contributions. We introduce the first style transfer method capable of transferring diverse style characteristics between 3D shapes, a task no previous method was capable of achieving. Our approach is based around the shape analogy metaphor developed in IQ tests. Our main technical contribution is a new algorithm for computing and assembling shape analogies suitable for graphics applications in a manner consistent with human logic, as reflected by these tests, leading to visually appealing results. We further demonstrate that our shape analogy framework can be used as-is to successfully complete partial 3D scans with the help of a user provided structural template, coherently propagating scan style across the completed surfaces, improving upon the state of the art in scan-completion.

\section{Related Work}

Structure-aware geometry processing and shape synthesis is an increasingly popular topic in computer graphics, see [MWZ*13] for a recent review. Below we discuss the works most relevant to our framework.

Shape Analogy Queries: Shape analogy queries have been studied extensively in cognitive science, see [SGKK09] and the references there in. Our work is inspired by these studies and aims to apply the analogy metaphor to practical styletransfer problems. Artificial intelligence research proposes several symbolic solutions to procedurally described shape analogy queries [Eva68, BO00]. In contrast, our method operates on non-parametric shape representations in an openworld setup, a significantly harder problem.

Shape Analysis: In recent years, many techniques have 
been proposed to detect the symmetric/repetitive geometric patterns on models and extract self-similarity transformations,e.g. [TW05, MGP06, PMW*08, MBB10]. Symmetry has been used to benefit diverse geometry processing applications [MWZ*13, PLR98]. Multiple approaches exist to compute full or partial mappings between similar shapes [SAPH04, vKZHCO11, KLM*12, $\left.\mathrm{HZG}^{*} 12, \mathrm{SNB}^{*} 12\right]$. In particular $\left[\mathrm{ROA}^{*} 13\right]$ use the notion of shape analogies to retrieve an output shape from a given database that relates to the exemplar like the target relates to the source. The source-to-target relationships they handle are expected to be continuous difference maps, a constraint unsuitable for style transfer between shapes with different structure. Our method builds upon this body of work, using analogy computation to guide the synthesis of new models. Our main challenge, not addressed by previous work, is to compute the best set of patch-wise transformations describing the source to target relationship in a style-transfer setting.

Part-based Shape Synthesis: Our output models are constructed by assembling patches from the exemplar (and target) models. The procedure used by our assembly process is very different from that of previous part-based modeling frameworks. Many earlier methods rely on users to specify and edit the assembled parts [FKS* 04, CKGK11]. Others rely on random or user-assisted derivations from shape grammars learned from a single exemplar [BWS10,TYK*12]. Finally, there are methods that automatically recombine interchangeable meaningful parts from large databases of models [KCKK12, XZCOC12, SFCH12]. In contrast, we aim to assemble the output model by automatically discovering and replicating the patch-wise analogy transformations between the source and target. We note that the patches we seek often do not correspond to model parts as defined in earlier work (e.g. Figures 4 or 6 ) but rather follow from the structural differences between the source and target.

Deformation Transfer: Methods, such as [SP04] are designed to transfer local pose information between models with similar structures leveraging a structure preserving nearcontinuous map between them. Consequently, they are not designed to perform one-to-many mapping of structural elements, e.g. map one pillow to two (see Figure 9, target 2). The source-to-target analogies we compute are designed to relate shapes with drastically different structures and capture structural differences rather than local shape variation. They therefore support the use of discontinuous per-patch correspondences, automatically computing one-to-many as well as one-to-none matches.

2D Style Transfer: The problem of style transfer for 2D curves has been explored in several pioneering works [HJO*01, HOCS02, FTP03]. The shape primitives and style representations they propose have limited scope and can not be applied to more general settings, especially when dealing with 3D shapes. For example, the curve analogies method of Hertzmann et al. [HOCS02] constrains the sourceto-target analogies to continuous, arc-length parametrized 'filter' transformations. This approach is not applicable to the many setups handled successfully by our framework,

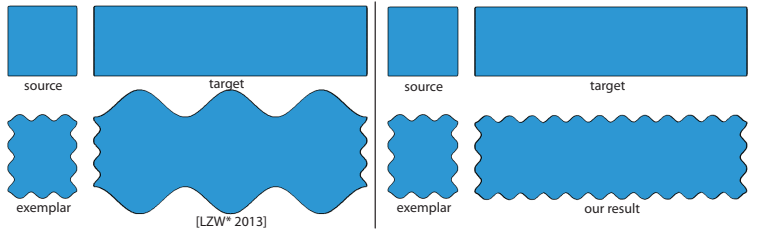

Figure 5: Left: Segment-based style transfer [LZW*13] considers only segment-level source to target transformations, leading to unnatural results. Right: Our shape analogy method operates at a finer, point-level granularity producing a more natural result.

where different partial, patch-wise, transformations need to be combined together to generate the desired analogy (e.g. Figure 8). More importantly, the notion of arc-length parameterization is 2D-curve specific and has no obvious 3D counterpart. The method of [FTP03] has a similar limitation. Li et al. [LZW*13] use feature-point based segmentation and segment-level correspondences to assemble a source-to-target analogy. This approach fails when segment-level resolution is too coarse to capture the source-to-target relationships (Figure 5). Feature-point based segmentation has no 3D surface equivalent, and generating meaningful segmentations of $3 \mathrm{D}$ shapes is a problem in its own right. By operating at a finer point-level granularity our method generates more intuitive solutions on 2D inputs (Figure 6) and, more significantly, seamlessly extends to the $3 \mathrm{D}$ domain.

3D Part-Scale Transfer: Xu et al. [XLZ*10] automate part-scale transfer, a special case of style-transfer, in 3D. Given a pre-defined coarse segmentation of the exemplar and target into one-to-one corresponding parts they use these correspondences to anisotropically scale exemplar parts to match the proportions of the matching parts on the target. The method has limited applicability, as it assumes one-to-one part level correspondence between the exemplar and target and cannot handle style properties beyond scale. We transfer a wide range of style properties between structurally different shapes by building source-to-target analogies which consists of multiple patch-wise transformations (Figures 1, 4). See Section 7 for more examples.

\section{Overview}

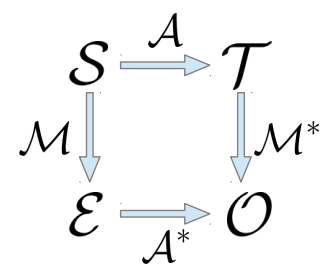

Figure 7: Analogy relationships. analogy transformations $\mathcal{A}$ and $\mathcal{A}^{*}$, relating the source to the target and the exemplar to the output respectively, are expected to be very similar (while effectively identical in the analogy query setup, they may slightly differ for real-life style transfer scenarios). To perform the style transfer we first recover the relationships $\mathcal{M}$ and $\mathcal{A}$ between the input models 


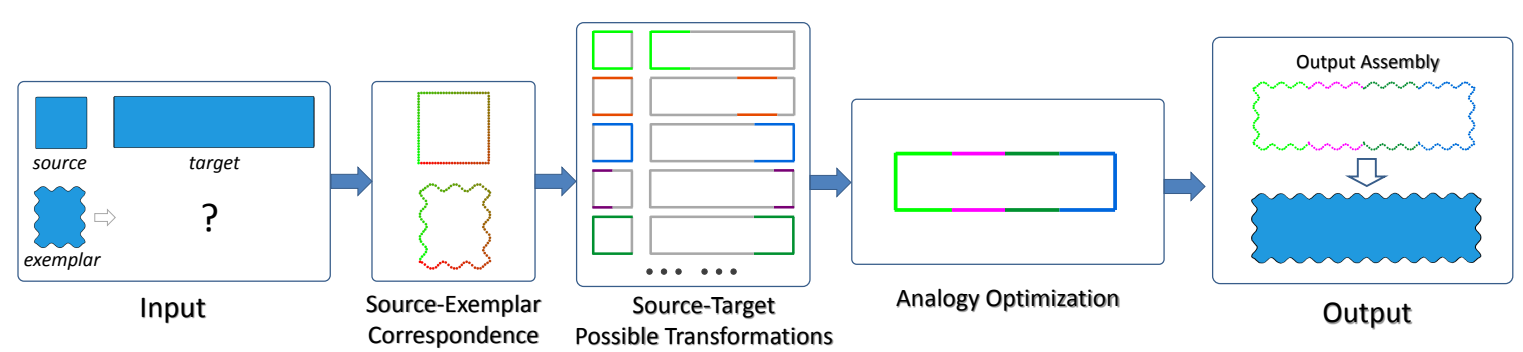

Figure 6: Overview of our method using 2D curve style transfer as an example. We first sample the input curves by uniformly distributed points, then compute the exemplar-source correspondence (color encodes matched points). Next, we compute candidate source-to-target transformations (color encodes matched patches). We use multi-label optimization to compute the best source-to-target analogies, from which we assemble the final output using the exemplar patches.

indicated by the arrows in the diagram (Sections 4 and 5). Assuming that, as in the traditional shape analogy quiz setup, $\mathcal{M}^{*}$ is similar to $\mathcal{M}$, we then approximate the exemplar to output transformation $\mathcal{A}^{*}$ by $\mathcal{M} \mathcal{A} \mathcal{M}^{-1}$, and further refine it using a simultaneous alignment and deformation process to obtain the desired output model (Section 6).

We expect the source-exemplar mapping $\mathcal{M}$ to be dense and continuous and compute it as described in Section 4. To compute the source to target analogy $\mathcal{A}$, we first extract the set of all plausible similarity transformations between the source and the target. This computation typically results in multiple alternative transformations for each source or target sample points. To assemble an analogy, we select the most suitable transformation for each target sample using a multi-label optimization procedure that balance our criteria of simplicity, compactness and output continuity (Section 5). In some cases, a target sample may have no corresponding transformation or source sample (e.g. the 'hat' above the circle in Figure 3 ). To handle such cases, we use a self-referencing transformation, which transfers target geometry to the output as-is.

Pre-Processing Our framework is designed to work on a variety of data sources, including scanned point clouds, meshes, and polygon soups. To unify the handling of heterogeneous data we represent all input shapes using pointsample sets. Specifically, for each input model we create a point set $\{s\}$ by uniformly sampling the model surface via Poisson Disk sampling. The sampling density reflects the level of detail of the input models. In addition, we make sure that the number of source samples is no less than the number of exemplar samples. For each sample $s$, we store its position $\mathbf{p}(s)$ and normal $\mathbf{n}(s)$. We assume all input models are consistently scaled and aligned.

\section{Source-Exemplar Correspondence}

Given a source and an exemplar model, we search for dense point-to-point correspondences between them. This is a problem that had been extensively investigated, see [SPR06, vKZHCO11] for related surveys. Our solution follows the spirit of the correspondence methods of [ACP03, SP04] aiming for correspondences that minimally deform the exemplar when mapping it to the source.

We leverage the initial compatible alignment of the two models and initialize the correspondence by computing a point-to-point exemplar to source matching that minimizes the sum of squared distances between the paired samples. We define a pair-wise distance between a source sample $s_{s}$ and an exemplar sample $s_{e}$ using their positions and normals:

$$
d\left(s_{S}, s_{e}\right)=\left|\hat{\mathbf{p}}\left(s_{S}\right)-\hat{\mathbf{p}}\left(s_{e}\right)\right|^{2}+\lambda \cdot \cos ^{-1}\left(\mathbf{n}\left(s_{S}\right) \cdot \mathbf{n}\left(s_{e}\right)\right)
$$

where $\hat{\mathbf{p}}$ is the relative position of each sample with respect to the bounding box of the shape it belongs to. $\lambda$ is set to the average squared distance between neighboring sample points. This minimization can be formulated as an assignment problem, efficiently solved via the Hungarian algorithm [Kuh55]. We then iteratively improve the correspondences, alternating between an assignment step and a deformation step that follows Sumner et al. [SP04]. Since the models are a priori reasonably well aligned, we typically require only a handful of iterations to converge. Finally, we ensure that each source point has a corresponding exemplar one (recall that the source sampling is enforced to be at least as dense as that of the exemplar). Given an unmatched source sample, we find its nearest matched sample on the source. We then match it to the corresponding exemplar sample of this matched neighbor. See Figures 6 and 9 for matching visualizations in $2 \mathrm{D}$ and $3 \mathrm{D}$.

We support manual specification of sparse pair-wise correspondences between samples on the two models to guide the matching. In this case we use the correspondences as constraints for the deformation step above. See Figure 8 for an example.

\section{Source to Target Analogy Assembly}

After computing the source-exemplar correspondence $\mathcal{M}$, our algorithm proceeds with the assembly of the analogy $\mathcal{A}$, the key technical component of our method. We first compute an initial large set of candidate patch-wise transformations (Section 5.1). We then employ a labeling step to assign each target sample the best possible transformation in terms of the criteria in Section 1 (Section 5.2).

\subsection{Transformation Selection}

To compute the initial candidate set of transformations, we consider all pairs of one source and one target samples, and have each pair vote for a transformation that best aligns their 
local coordinate systems. The local coordinate system of each point is determined by its normal and its principal curvature directions. These directions are optimized to "fill in" the curvature field in areas where it is poorly defined (umbilical regions) based on the optimizing technique of [HZ00]. Our subsequent clustering step filters out any local noise that may be present in this raw data.

Following [MGP06], each pair of local coordinate systems define a unique rotation, translation, and uniform scale, thus forming transformation votes in a six-dimensional space. We perform mean-shift clustering of the votes to find the cluster centers indicating the strongest modes of source-to-target transformations.

The result of this procedure is an initial candidate set of transformations $\mathcal{Q}$ corresponding to the detected modes (cluster centers) in the above transformation space. Each transformation $\mathbf{Q} \in \mathcal{Q}$ is associated with a subset of samples on the target surface $\left\{s_{t}\right\} \subset \mathcal{T}$, where each target sample $s_{t}$ can be matched to a source sample $s_{s} \in \mathcal{S}$ with consistent surface normal and principal curvature magnitudes under the transformation $\mathbf{Q}$ :

$$
\left|\mathbf{Q}\left(\mathbf{p}\left(s_{t}\right)\right)-\mathbf{p}\left(s_{s}\right)\right|<\delta_{\mathbf{p}}, \mathbf{Q}\left(\mathbf{n}\left(s_{t}\right)\right) \cdot \mathbf{n}\left(s_{s}\right)>\delta_{\mathbf{n}}
$$

Here $\delta_{\mathbf{p}}=2 \alpha$ where $\alpha$ is the average distance of source and target samples to their nearest neighboring sample and $\delta_{\mathbf{n}}=0$ (i.e., maximum accepted angle difference between normals is 90 degrees).

For target samples not associated with any transformation, we add to the candidate set the self-referencing transformation, mapping the sample to itself.

\subsection{Analogy Optimization}

For most target samples the candidate set of transformations $\mathcal{Q}$, described above, contains multiple alternative transformations. To assemble the desired analogy, we perform an optimization step to obtain a compact subset of transformations and their associated regions of influence on the target shape that are as simple as possible and preserve continuity on the output model.

We formulate the analogy computation as a multi-label optimization problem. Each transformation in our candidate set is associated with a label. Our goal is to compute the most probable configuration of labels on the target surface so that each target sample $s_{t}$ is associated with the most plausible onto transformation (label) from our candidate set. To compute the labels we minimizing the following objective function:

$$
\begin{aligned}
E\left(\mathbf{C} ;\left\{s_{t}\right\}\right)= & \sum_{t} E_{1}\left(C_{t} ; s_{t}\right)+\sum_{t, t^{\prime} \in \mathcal{N}(t)} E_{2}\left(C_{t}, C_{t^{\prime}} ; s_{t}, s_{t^{\prime}}\right) \\
& +\sum_{\mathbf{Q}_{l} \in \mathcal{Q}} E_{3, \mathbf{Q}_{l}}(\mathbf{C})
\end{aligned}
$$

where $\mathbf{C}=\left\{C_{t}\right\}$ is the set of random variables representing the label per target sample $s_{t} . E_{1}$ is an unary energy term that assesses the consistency of each target sample with each label individually, evaluating how well the transformation aligns the particular sample with the source surface. $E_{2}$ is the pairwise energy term that measures the consistency of joint label assignments to pairs of neighboring target samples $\left(s_{t}, s_{t}^{\prime}\right)\left(t^{\prime} \in \mathcal{N}(t)\right.$ are the neighboring points of $\left.s_{t}\right)$, promoting compactness. Lastly, $E_{3, \mathbf{Q}_{l}}$ is a regularization term which gives preference to simpler transformations and penalizes the existence of each individual transformation $\mathbf{Q}_{l}$ in the output analogy, thus forcing the solution to have as few unique transformations as necessary. Below we explain our formulation for each energy term.

Unary term The unary term evaluates the probability of a target sample $s_{t}$ belonging to each of the candidate transformations. The probability per label is converted to an energy value through its negative logarithm:

$$
E_{1}\left(C_{t} ; s_{t}\right)=-\log P\left(C_{t} \mid s_{t}\right)
$$

The above conditional probability is evaluated as follows:

$$
\begin{aligned}
P\left(C_{t}=l \mid s_{t}\right) \propto & \exp \left\{-\frac{\left\|\mathbf{Q}_{l}\left(\mathbf{p}\left(s_{t}\right)\right)-\mathbf{p}\left(s_{s}\right)\right\|^{2}}{2 \sigma_{\mathbf{p}}^{2}}\right\} . \\
& \exp \left\{\frac{\mathbf{Q}_{l}\left(\mathbf{n}\left(s_{t}\right)\right) \cdot \mathbf{n}\left(s_{s}\right)}{\sigma_{\mathbf{n}}^{2}}\right\}
\end{aligned}
$$

where $\mathbf{Q}_{l}$ is the transformation associated with label $l$, and $s_{S}$ is the closest compatible point on the source shape for the target sample $s_{t}$ under the transformation $\mathbf{Q}_{l}$. Here, the first factor uses a Gaussian function on distances between samples, while the second factor adopts an angular Gaussian function (as used in the von Mises distribution) on angles between normals. The larger the distance (or the angular distance of normals) between the target sample and its nearest corresponding source point under the transformation $\mathbf{Q}_{l}$, the smaller the probability is for assigning that label $l$ to this target sample. The standard deviation parameters are set to $\sigma_{\mathbf{p}}=0.1 \alpha$ ( $\alpha$ is the average distance of source and target samples to their nearest neighboring sample), and $\sigma_{\mathbf{n}}=0.1(\pi / 2)$. The above unary term probabilities are normalized so that their sum over the labels per sample is equal to one.

Pairwise term The pairwise term penalizes neighboring samples being assigned different labels $\left\{l, l^{\prime}\right\}$, minimizing the number of output transformations and preferring more compact labelings with shorter boundaries. We consider a target sample to be a neighbor to another sample, if the geodesic distance between them is less than $5 \alpha$ and their normals are compatible (i.e., differ less than 90 degrees). The pairwise term is expressed as follows:

$$
\begin{aligned}
E_{2}\left(C_{t} \quad\right. & \left.=l, C_{t^{\prime}}=l^{\prime} ; s_{t}, s_{t^{\prime}}\right)= \\
& {\left[l \neq l^{\prime}\right]\left(-\log P\left(C_{t}=l, C_{t^{\prime}}=l^{\prime} \mid s_{t}, s_{t^{\prime}}\right)+\mu\right)(6) }
\end{aligned}
$$

where the $\mu$ parameter penalizes the length of boundaries between regions with different labels [KHS10]. In our experiments, we set $\mu=0.5$. The term $P\left(C_{t}=l, C_{t^{\prime}}=l^{\prime}\right.$ $\left.s_{t}, s_{t^{\prime}}\right)$ expresses the probability for assigning the labels $\left\{l, l^{\prime}\right\}$ to the neighboring samples $\left\{s_{t}, s_{t^{\prime}}\right\}$. This term also favors continuity on the output shape when the corresponding transformations $\left\{\mathbf{Q}_{l}, \mathbf{Q}_{l^{\prime}}\right\}$ are applied to these neighboring 
samples:

$$
\begin{aligned}
& P\left(C_{t}=l, C_{t^{\prime}}=l^{\prime} \mid s_{t}, s_{t^{\prime}}\right)=1.0- \\
& \exp \left\{-\frac{\left\|\left[\mathbf{p}\left(s_{o, \mathbf{Q}_{l}}\right)-\left(\mathbf{p}\left(s_{O^{\prime}}, \mathbf{Q}_{l^{\prime}}\right)\right)\right]-\left[\mathbf{p}\left(s_{t}\right)-\mathbf{p}\left(s_{t^{\prime}}\right)\right]\right\|^{2}}{2 \sigma_{\mathbf{p}}^{2}}\right\} . \\
& \exp \left\{\frac{\cos (\theta-\phi)}{\sigma_{\mathbf{n}}^{2}}\right\}
\end{aligned}
$$

where $s_{o, \mathbf{Q}_{l}}$ is the output sample produced by applying the transformation $\mathbf{Q}_{l}{ }^{-1} M \mathbf{Q}_{l}$ to the target sample $s_{t}$, and similarly $s_{O^{\prime}, \mathbf{Q}_{l^{\prime}}}$ is the output sample produced by applying the transformation $\mathbf{Q}_{l^{\prime}}{ }^{-1} M \mathbf{Q}_{l^{\prime}}$ to the target sample $s_{t^{\prime}}$. The angle $\phi$ is the angle between the normals of the target samples $s_{t}$ and $s_{t^{\prime}}$ and $\theta$ is the angle between the normals of the corresponding output samples.

The factors in this term follow the Gaussian functions as above. The probability for assigning a pair of labels $\left\{l, l^{\prime}\right\}$ to neighboring target samples is higher when the position and normal differences of the resulting output samples is similar to the ones of the corresponding target samples after applying the corresponding transformations $\left\{\mathbf{Q}_{l}, \mathbf{Q}_{l^{\prime}}\right\}$, promoting output continuity. The standard deviation parameters have the same values as the ones in the unary term.

Regularization term The regularization term penalizes solutions based on the set of labels appearing in them, encoding a preference for explaining the structural differences between the source and target with as few transformations as necessary, further reinforcing compactness. In addition, the term encodes a preference for simpler transformations including the identity transformation $\mathbf{I}$, translations, and rotations with angles $\kappa(\pi / 2)$ ( $\kappa$ is an integer) along the $x$ ,$y$-, $z$-axes. Following [DOIB12], we introduce label costs in this regularization term:

$$
E_{3, \mathbf{Q}_{l}}(\mathbf{C})=\left\{\begin{array}{l}
-N \cdot \log P\left(E_{l}=1\right) \text { if } \exists t: C_{t}=l \\
0 \text { otherwise }
\end{array}\right.
$$

where $E_{l}$ is a binary variable representing whether the transformation $\mathbf{Q}_{l}$ is used, and $N$ is the number of target samples. The probability that the transformation $\mathbf{Q}_{l}$ is used is equal to 1 when $l$ corresponds to the identity transformation. For the rest of the transformations:

$$
\begin{aligned}
P\left(E_{l}=1\right)=0.5 \cdot & \frac{\exp \left(\cos \left(4 R_{l, x}\right)\right)}{\exp (1)} \cdot \frac{\exp \left(\cos \left(4 R_{l, y}\right)\right)}{\exp (1)} . \\
& \frac{\exp \left(\cos \left(4 R_{l, z}\right)\right)}{\exp (1)} \cdot \exp \left(-\left(s_{l}-1\right)^{2}\right)(9)
\end{aligned}
$$

where $R_{l, x}, R_{l, y}, R_{l, z}$ are the rotation angles along the $x$-, $y$ , $z$-axis respectively, and $s_{l}$ is the uniform scale associated with the transformation $\mathbf{Q}_{l}$. Transformations with rotations angles $0,90,180$, or 270 degrees and with unit scale have $50 \%$ probability. Otherwise, their probability decreases.

Optimization The above objective function $E\left(\mathbf{C} ;\left\{s_{t}\right\}\right)$ is optimized using graph-cuts with label costs [DOIB12]. Although the algorithm is not guaranteed to find a global minimum (since the problem is NP-hard [DOIB12]), it practically reaches a good solution with low total energy value. The solution implicitly defines a segmentation of the target shape into labeled regions - each region is associated with a transformation $\mathbf{Q}_{l}$ based on its estimated label $l$. Due to the greedy nature of the optimizer, the estimated labeling may sometimes contain isolated small patches of points with different label from their neighbors. In a post-processing step, our algorithm finds target samples whose label disagrees with more than $90 \%$ of the labels assigned to their neighbors inside the above defined geodesic rings, and switches their label to the most common label inside these geodesic rings.

\section{Output Synthesis}

Given the analogy transformation $\mathcal{A}$ and the source-toexemplar mapping $\mathcal{M}$, we first approximate the exemplarto-output transformation $\mathcal{A}^{*}$ by applying $\mathcal{M} \mathcal{A} \mathcal{M}^{-1}$ to each exemplar sample independently. The resulting outputs are typically continuous within the source patches operated on by the analogies, but may exhibit discontinuities along boundaries, as real data doesn't perfectly comply with the strict shape analogy assumptions. We eliminate the discontinuities through a combination of rigid transformations and local deformation. We first extend each patch to generate overlapping regions by adding to each the samples in the geodesic neighborhood of the patch boundary samples. We then locate all sample pairs across different patches which after applying the associated transformations, are placed close to one another in terms of positions and normals. Our deformation framework aims to align the patches so as to minimize the distance between these samples along the normal direction. To preserve patch geometry, we also consider pairs of adjacent samples inside each patch and seek to maintain their relative locations. We formulate patch alignment as a least-squares problem and simultaneously solve for the positions of all the output samples by minimizing the following energy function:

$$
\begin{aligned}
E\left(\left\{\mathbf{p}\left(s_{o}\right)\right\}\right) & =\sum_{m_{o} \neq m_{o^{\prime}}}\left[\left(\mathbf{n}\left(s_{o}\right)+\mathbf{n}\left(s_{O^{\prime}}\right)\right) \cdot\left(\mathbf{p}\left(s_{o}\right)-\mathbf{p}\left(s_{o^{\prime}}\right)\right)\right]^{2} \\
& +\sum_{m_{o}=m_{o^{\prime}}}\left\|\left(\mathbf{p}\left(s_{o}\right)-\mathbf{p}\left(s_{o^{\prime}}\right)\right)-\left(\hat{\mathbf{p}}\left(s_{o}\right)-\hat{\mathbf{p}}\left(s_{O^{\prime}}\right)\right)\right\|^{2} \\
& +\mathrm{v} \cdot \sum_{o}\left\|\left(\mathbf{p}\left(s_{o}\right)-\hat{\mathbf{p}}\left(s_{o}\right)\right)\right\|^{2}
\end{aligned}
$$

where $s_{O}$ and $s_{o^{\prime}}$ are adjacent samples, $\mathbf{p}\left(s_{o}\right)$ are the sample positions to be determined, $\hat{\mathbf{p}}\left(s_{o}\right)$ are the sample position before alignment, and $m_{o}$ and $m_{o^{\prime}}$ are the patch segments associated with $s_{o}$ and $s_{o^{\prime}}$ respectively. The first term aims to align nearby samples on adjacent patches while the second aims to preserve the relationships between adjacent points in the same patch. These two terms alone yield an under-defined system as they only involve relative locations, allowing the solution to translate arbitrarily. To stabilize the solution, we add a third term aiming to minimize the changes in the sample positions assigning it a very small weight $v=0.01$. Minimizing this quadratic functional amounts to solving for the positions that satisfy $\nabla E\left(\mathbf{p}\left(s_{i}\right)\right)=0$, a standard linear system solved using Modified Cholesky Decomposition. The input and output of this stage are shown in Figure 13e. 


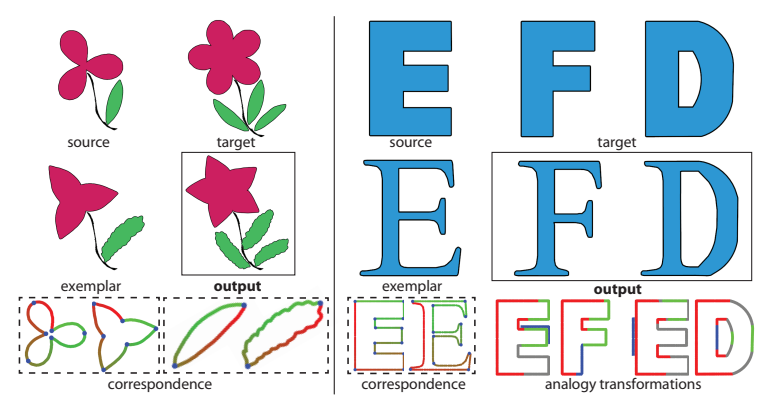

Figure 8: $2 D$ style transfer.

Post-Processing When the input exemplar is provided as a triangle mesh, we aim for a similarly defined output model. Reconstructing the output from the sample points would not achieve this goal as it will not preserve exemplar features. To transfer the exemplar triangulation, we first associate each exemplar triangle with the closest exemplar sample. For each synthesized output sample $s_{o}$, we track the corresponding exemplar sample $s_{e}$ and apply the sample transformation to all the triangles associated with it. The result of this step is an imperfectly aligned triangle soup. To improve alignment we we minimize the energy described by Equation 10, this time with mesh vertex positions as variables. The result is not guaranteed to be a closed mesh, but neither are most of our inputs.

\section{Results and Applications}

Throughout the paper we show numerous examples of style transfer performed using our framework.

2D Style Transfer. To compare our results against previous 2D curve style transfer approaches and to validate the analogy assembly criteria we used we applied the method to a number of 2D inputs with varying complexity (Figures 2, 6 and 8). Figure 2 mimics a classical analogy IQ test input. Figure 8 (left) shows an example of transferring the drawing style of a flower. Note the different patch-wise transformations applied to the petals and leaves. Figure 8 (right) shows a challenging example of transferring a font style from the letter ' $E$ ' to two other letters. While the ' $F$ ' may appear like a simple subset of ' $\mathrm{E}$ ', the challenge is to complete its leg realistically and maintain the collinearity along it. The ' $\mathrm{D}$ ' is particularly challenging as it contains elements not present in the sourceexemplar pair. Our method accomplishes the task using a set of transformations that includes self-referencing, indicated with gray color in the color-coded analogy transformations visualization (Figure 8).

The transfers we have accomplished cannot be computed by earlier methods. Arc-length parameterization based approaches [HOCS02, FTP03] would require the user to specify the pairs of matching curve segments manually for each input. While the more recent approach of [LZW*13] may succeed on inputs such as the flower or letters (Figures 8) with a sufficiently fine-tuned segmentation step, it fails on inputs like the square-to-rectangle style transfer in Figure 5 where the patches defining the analogy do not naturally align with segments on the source and target models. In contrast our method is able to produce the expected result.

3D Style Transfer. The 3D soft-furniture results (Figures 1,9$)$ demonstrate style transfer for complex 3D shapes. As highlighted by the analogy visualizations (Figure 9, 4), the transformations required for relating the input sources and targets are quite complex, containing a mixture of translations and rotations. The assembled analogies are successfully used to generate two series of furniture from two different exemplars. Our method is the first to transfer style properties that go beyond part scale ( [XLZ*10]) in 3D (see Figure 10). Below we show more examples of applying our style-transfer technique in the context of different applications.

Computation Time. The 2D results shown in this paper are all generated within seconds. The computation time for 3D results, depending on input complexity, ranges from seconds to several minutes on a PC with Intel Core-i7 3.2GHz CPU and $8 \mathrm{~GB}$ memory. See Table 1 for typical statistics.

In addition to style transfer our framework can be used, with minor changes, for template based scan-completion and style exchange.

Scan Completion. Completion methods aim to plausibly fill in the missing regions in input partial range scans. When the holes are large (e.g. Figure 13) a common approach is to employ a template shape whose structure is similar to the scan to aid the completion process [KS05, $\left.\mathrm{PMG}^{*} 05\right]$. These methods compute a mapping between the partial scan and the template shape, and then blend them together, generating hybrid models that fill the gaps and holes in the scan with suitably deformed template geometry. While the results have the overall desired structure, they do not account for fine-level differences between the exemplar and the template (Figure 11, mid-right). Specifying high level template symmetries $\left[\mathrm{PMG}^{*} 05\right]$ can improve the results, but cannot account for more complex self-similarities. With minimal changes, our framework can be used to improve completion results, propagating scan details and style across the completed areas (Figure 11, right). To solve scan completion via the shape analogy framework, we first compute a mapping from the scan to the template, and then treat the scan as the exemplar, the image of the computed mapping on the template as the source, and the entire template as the target. The rest of the processing remain unchanged.

We tested our framework on several complex partial scans. The chair and arc (Figure 12) showcase our method on singleview scans generates using a synthetic scanner. The input exemplars consequently lack all the back-facing and occluded surfaces. For the chair we miss its backside, the bottom of the seat and back sides of all four legs. The template chair we use has different leg shape (square instead of round) and different back dimensions. The completion uses only data present in the scan itself, without blending in any template geometry. To control for the difference in chair back dimensions, we specify four pairs of point-to-point correspondences between the chair backs - two on the top corners and two on the bottom corners as input to help the correspondence computation in 

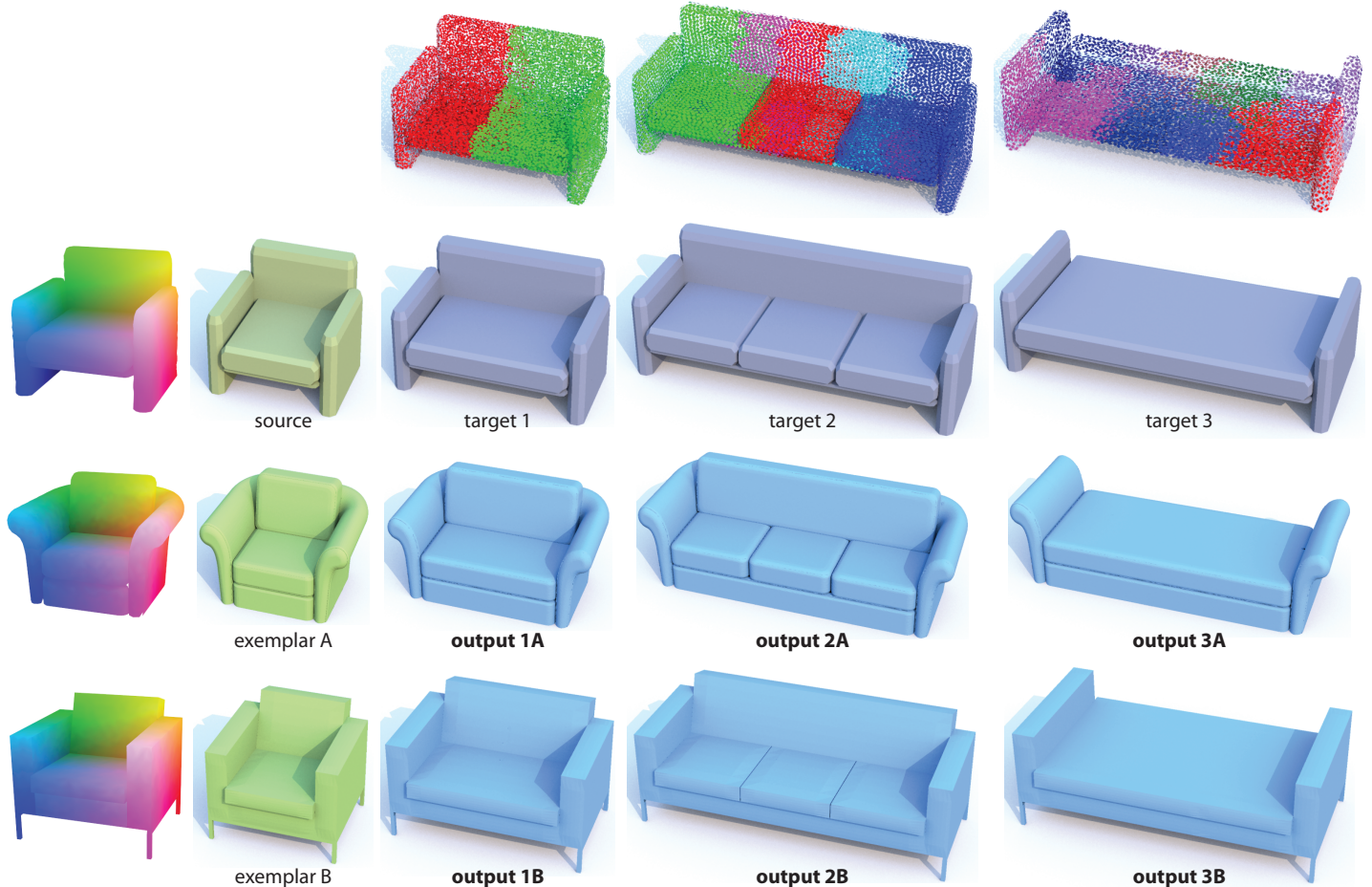

Figure 9: Style transfer for soft furniture. Given a source armchair and a series of other soft furnishings as targets, we successfully transfer two different exemplar styles to each item in the series, a task that would be tedious and cumbersome to perform manually. Top row shows the analogy transformations for each computed target. Left column shows the source-exemplar correspondences.

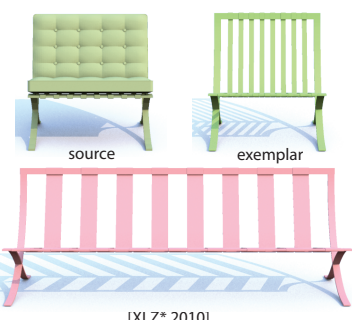

$\left|X L \iota^{*} 2010\right|$
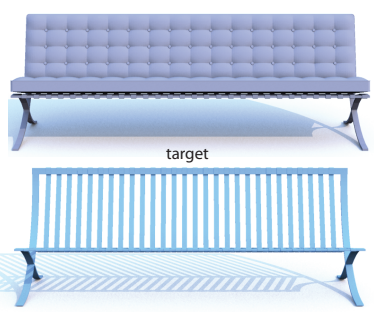

our result

Figure 10: Comparison with anisotropic part scaling.

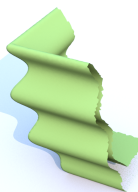

exemplar

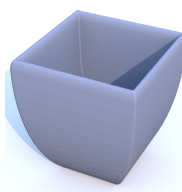

template

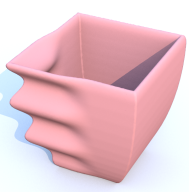

blending result

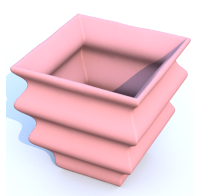

our result

Figure 11: Template-based scan completion. Left to right: partial scan, input template, scan-template correspondence followed by blending [KSO5], and our result.

Section 4 . The template for the arc is very simple compared to the scan. Using the assembled analogy we are able to convincingly complete both scans, replicating scan style details across the completed regions.

The last example, a vase shown in Figure 13, is particularly challenging. Here the scanned data come from a commercial hand-held scanner and consist of two small vase fragments. Given a basic cylinder as a template, our method

automatically recovers the analogy between the fragment's image on the cylinder and the entire cylinder and applies it to the exemplar, generating a convincing output.

Style Exchange. In some situations, a user may want to transfer a style from an exemplar to a target, but may not have an appropriate source model, facilitating style-structure separation. While shape analogies are ill-defined when no source is provided, Figure 14 demonstrates a user-assisted variation of our method that successfully tackles this setup through the use of box-like source/target models computed semi-automatically. In both figures, the input models are three exemplars shown in green and our goal is to exchange their styles with the other inputs. We first segment the exemplar models into meaningful components, and compute the axis-aligned bounding box of each component. We use the combination of the boxes (shown in the first row) as either source or target depending on the context. Specifically, for each green exemplar model, the corresponding blue model above in the first row is the source, and the other two blue models serve as the targets. With this box representation, the candidate set of transformations can be reduced to all possible transformations between pairs of boxes, including translation, rotation and non-uniform scaling. From this set of candidate transformations, our analogy assembly algorithm automatically computes the optimal box-wise transformations between the source and target models. This construction leads to the desired style transfer results, shown in light blue. 

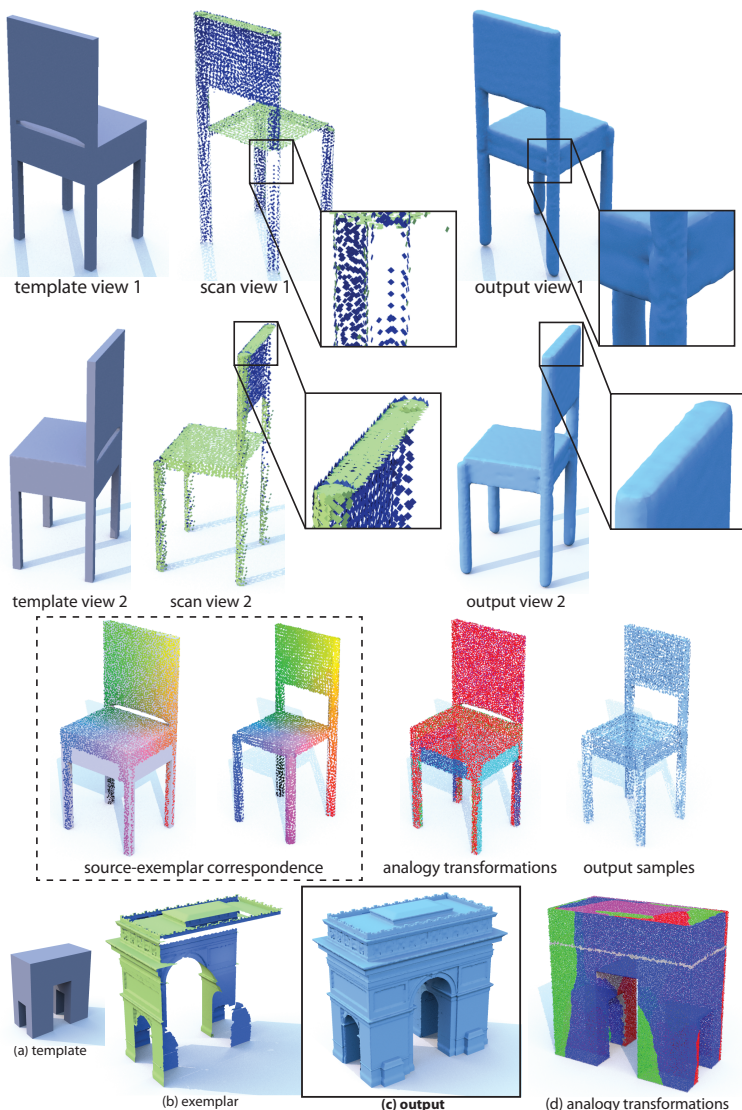

Figure 12: Scan completion of single-view chair and arc scans. Both templates have very different style from the scanned models (e.g., different leg shape). The zoomed-in views on the right show details of the scanned data and the reconstructed model.

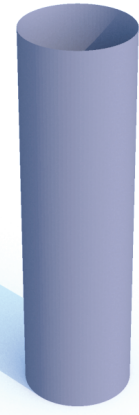

(a) template

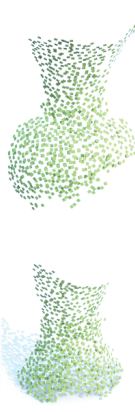

(b) exemplar

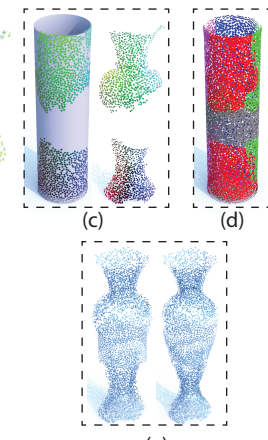

(e)

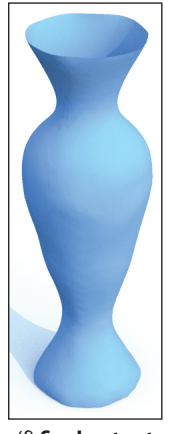

(f) final output
Figure 13: Scan completion of a vase: Exemplar to template mapping shown in (c) - the image of the mapping defines the source; $(d)$ computed analogy transformations (identity in red, self-similarity in gray); (e) output samples before and after alignment; (f) final reconstructed output.

\section{Conclusions}

We presented a new style transfer method suitable for both 2D and 3D data. While previously work on 3D styles was limited
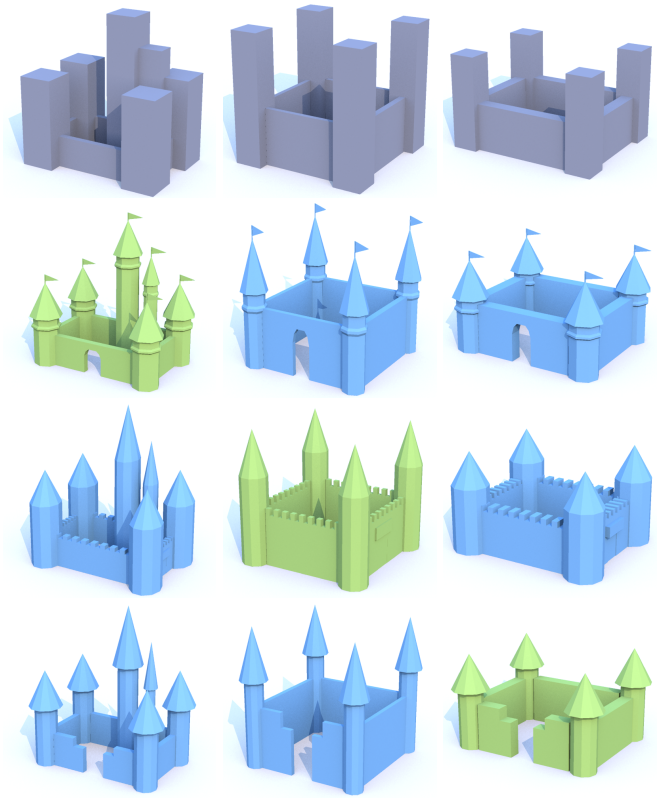

Figure 14: Style exchange between a set of castles.

\begin{tabular}{|c|c|c|c|c|c|}
\hline \multirow{2}{*}{ demo } & \multicolumn{4}{|c|}{ number of samples } & \multirow{2}{*}{$\begin{array}{c}\text { runtime } \\
\text { (min) }\end{array}$} \\
\hline & source & target & exemplar & output & \\
\hline corner sofa & 7600 & 14566 & 3938 & 14373 & 3 \\
\hline chair & 2781 & 11740 & 3577 & 8659 & 1 \\
\hline arc & 10442 & 30543 & 11974 & 41546 & 5 \\
\hline vase & 1094 & 5206 & 1739 & 4803 & 1 \\
\hline
\end{tabular}

Table 1: Typical algorithm statistics.

to transferring parts scales between co-segmented shapes [XLZ* 10], our method is capable of capturing fine-level style features, such as part shapes or geometric textures and can process source and target models with different structure. We achieve our goal by computing source-to-target analogies which define in geometric terms the structural differences between them. Applying a computed analogy to the exemplar effectively substitutes is structure with that of the target, while retaining the exemplar details, achieving the desired style transfer effect.

Limitations and future work. In our current implementation, the per-patch transformations used to assemble the analogies are restricted to rigid similarity transformations. However, one can think of setups where the source and target are related via more complex, free-form deformations commonly found in organic shapes. It would be interesting to investigate how our method can be extended to handle these more general setups. In addition, our method assumes consistent alignment and orientations of the input models, a task that we currently do manually but that can be automated using shape matching and analysis tools. In some cases the shape analogy problem is ill-posed in that there may be multiple possible analogy transformations and thus multiple solutions. We are aimed at proposing one reasonable output and leave enumerating all the solutions as future work. Finally, the most intriguing future research direction is 
inferring a source shape given only the target and exemplar shape.

Acknowledgements We would like to thank Niloy Mitra and Richard Zhang for sharing source code, Daniel Cohen-Or for valuable comments, Liangliang Nan for scanned vase data, Mikhail Bessmeltsev and Andrew McGregor for help with images and videos. This work was partially funded by NSERC and GRAND NCE.

\section{References}

[ACP03] Allen B., Curless B., Popović Z.: The space of human body shapes: reconstruction and parameterization from range scans. ACM Trans. Graphics 22, 3 (2003), 587-594. 4

[BO00] Bohan A., O’Donoghue D.: LUDI: A model for geometric analogies using attribute matching. In Proc. Artificial Intelligence and Cognitive Science Conference (2000). 2

[BWS10] BoKELOH M., WAND M., SEIDEL H.-P.: A connection between partial symmetry and inverse procedural modeling. ACM Trans. Graphics 29 (2010), 104:1-104:10. 3

[CKGK11] Chaudhuri S., Kalogerakis E., Guibas L., KolTUN V.: Probabilistic reasoning for assembly-based 3D modeling. ACM Trans. Graphics 30, 4 (2011), 35:1-35:10. 3

[DOIB12] Delong A., Osokin A., IsACK H. N., BoYkov Y.: Fast approximate energy minimization with label costs. Int'l Journal of Computer Vision 96, 1 (2012), 1-27. 6

[Eva68] Evans T. G.: A program for the solution of a class of geometric-analogy intelligence-test questions. In Semantic Information Processing. Cambridge, Massachusetts, 1968. 2

[FKS*04] Funkhouser T., KaZhdan M., Shilane P., Min P., Kiefer W., TAL A., RUSinkieWICZ S., DobKIN D.: Modeling by example. ACM Trans. Graphics 23, 3 (2004), 652-663. 3

[FTP03] Freeman W. T., Tenenbaum J. B., Pasztor E. C.: Learning style translation for the lines of a drawing. ACM Trans. on Graphics 22, 1 (2003), 33-46. 3, 7

[HJO*01] HertzManN A., Jacobs C. E., Oliver N., Curless B., Salesin D. H.: Image analogies. In Proc. SIGGRAPH (2001), pp. 327-340. 1, 3

[HOCS02] Hertzmann A., Oliver N., Curless B., Seitz S. M.: Curve analogies. In Proc. Eurographics workshop on Rendering (2002). 3, 7

[HZ00] HERTZMANN A., ZORIN D.: Illustrating smooth surfaces. In Proc. SIGGRAPH (2000), pp. 517-526. 5

[HZG*12] HuAnG Q.-X., Zhang G.-X., GAO L., Hu S.-M., BUTSCHER A., GUIBAS L.: An optimization approach for extracting and encoding consistent maps in a shape collection. ACM Trans. Graphics 31, 6 (2012), 167:1-167:11. 3

[KCKK12] Kalogerakis E., Chaudhuri S., Koller D., Koltun V.: A probabilistic model for component-based shape synthesis. ACM Trans. Graphics 31, 4 (2012), 55:1-55:11. 3

[KHS10] Kalogerakis E., Hertzmann A., Singh K.: Learning 3D mesh segmentation and labeling. ACM Trans. Graphics 29, 4 (2010), 102:1-102:12. 5

[KLM*12] KiM V. G., Li W., Mitra N., DiVerdi S., FUNKHOUSER T.: Exploring collections of 3D models using fuzzy correspondences. ACM Trans. Graphics 31, 4 (2012), 54:154:11. 3

[KS05] Kraevoy V., Sheffer A.: Template-based mesh completion. In Proc. Symposium on Geometry Processing (2005) 7, 8

[Kuh55] KuHN H.: The hungarian method for the assignment problem. Naval research logistics quarterly (1955). 4
[LZW*13] Li H., Zhang H., WAng Y., CAO J., Shamir A., COHEN-OR D.: Curve style analysis in a set of shapes. Computer Graphics Forum (2013), To Appear. 2, 3, 7

[MBB10] Mitra N., Bronstein A., Bronstein M.: Intrinsic regularity detection in 3D geometry. In Proc. ECCV (2010), pp. 398-410. 3

[MGP06] Mitra N. J., Guibas L. J., Pauly M.: Partial and approximate symmetry detection for 3D geometry. ACM Trans. Graphics 25, 3 (2006), 560-568. 3, 5

[MWZ*13] Mitra N. J., Wand M., Zhang H., COHEN-OR D., BOKELOH M.: Structure-aware shape processing. In Eurographics State of the Art Reports (2013), pp. 175-197. 1, 2, 3

[PLR98] PotTMAnN H., LEE I., RANDRUP T.: Reconstruction of kinematic surfaces from scattered data. In Proceedings of symposium on geodesy for geotechnical and structural engineering (1998), pp. 483-488. 3

[PMG*05] Pauly M., Mitra N. J., Giesen J., Gross M., Guibas L. J.: Example-based 3D scan completion. In Proc. Symposium on Geometry Processing (2005), pp. 23-32. 7

[PMW* 08] Pauly M., Mitra N. J., Wallner J., Pottmann H., Guibas L. J.: Discovering structural regularity in 3D geometry. ACM Trans. Graphics 27, 3 (2008), 43:1-43:11. 3

[ROA*13] Rustamov R. M., Ovsjanikov M., AzenCot O. Ben-Chen M., Chazal F., Guibas L.: Map-based exploration of intrinsic shape differences and variability. ACM Trans. Graph 32, 4 (2013), 72:1-72:12. 3

[SAPH04] Schreiner J., Asirvatham A., Praun E., Hoppe H.: Inter-surface mapping. ACM Trans. Graphics 23, 3 (2004), 870-877. 3

[SFCH12] ShEn C.-H., FU H., CHEN K., Hu S.-M.: Structure recovery by part assembly. ACM Trans. Graphics 31, 6 (2012), 180:1-180:11. 3

[SGKK09] SChWERING A., GuST H., KÜHNBERGER K., KRUMNACK U.: Solving geometric proportional analogies with the analogy model HDTP. In Proc. Annual Meeting of the Cognitive Science Society (2009). 2

[SNB*12] Solomon J., NguYen A., Butscher A., BenChen M., Guibas L.: Soft maps between surfaces. Computer Graphics Forum 31, 5 (2012), 1617-1626. 3

[SP04] Sumner R. W., Popović J.: Deformation transfer for triangle meshes. ACM Trans. Graphics 23, 3 (2004), 399-405. 3, 4

[SPR06] Sheffer A., Praun E., Rose K.: Mesh parameterization methods and their applications. Foundation and Trends in Computer Graphics and Vision 2, 2 (2006), 105-171. 4

[TW05] Thrun S., WEgBREIT B.: Shape from symmetry. In Proc. ICCV (2005), pp. 1824 - 1831. 3

[TYK*12] TALTON J., YANG L., KumaR R., LiM M., GoOdMAN N. D., MĚCH R.: Learning design patterns with bayesian grammar induction. In Proc. UIST (2012), pp. 63-74. 3

[vKZHCO11] VAN KAICK O., ZHANG H., HAMARNEH G., COHEN-OR D.: A survey on shape correspondence. Computer Graphics Forum 30, 6 (2011), 1681-1707. 3, 4

[WXL*11] Wang Y., XU K., Li J., Zhang H., Shamir A., LiU L., Cheng Z., XIONG Y.: Symmetry hierarchy of man-made objects. Computer Graphics Forum 30, 2 (2011). 2

[XLZ*10] Xu K., Li H., Zhang H., Cohen-Or D., Xiong Y., CHENG Z.-Q.: Style-content separation by anisotropic part scales. ACM Trans. Graphics 29, 6 (2010), 184:1-184:10. 1, 3, 7, 9

[XZCOC12] Xu K., Zhang H., Cohen-Or D., Chen B.: Fit and diverse: Set evolution for inspiring 3D shape galleries. $A C M$ Trans. Graphics 31, 4 (2012), 57:1-57:10. 3 